\title{
Hierarchical MPC Secondary Control for Electric Power System
}

\author{
Freddy Milla, ${ }^{1,2}$ Manuel A. Duarte-Mermoud, ${ }^{1,2}$ and Noreys Aguila-Camacho ${ }^{1,2}$ \\ ${ }^{1}$ Department of Electrical Engineering, University of Chile, Avenue Tupper 2007, 8370451 Santiago, Chile \\ ${ }^{2}$ Advanced Mining Technology Center (AMTC), University of Chile, Avenue Beauchef 850, 8370451 Santiago, Chile
}

Correspondence should be addressed to Freddy Milla; fmilla@ing.uchile.cl

Received 13 December 2013; Revised 3 March 2014; Accepted 3 March 2014; Published 17 April 2014

Academic Editor: Hamid Reza Karimi

Copyright (C) 2014 Freddy Milla et al. This is an open access article distributed under the Creative Commons Attribution License, which permits unrestricted use, distribution, and reproduction in any medium, provided the original work is properly cited.

\begin{abstract}
Although in electric power systems (EPS) the regulatory level guarantees a bounded error between the reference and the corresponding system variables, to keep its availability in time, optimizing the system operation is required for operational reasons such as, economic and/or environmental. In order to do this, there are the following alternative solutions: first, replacing the regulatory system with an optimized control system or simply adding an optimized supervisory level, without modifying the regulatory level. However, due to the high cost associated with the modification of regulatory controllers, the industrial sector accepts more easily the second alternative. In addition, a hierarchical supervisory control system improves the regulatory level through a new optimal signal support, without any direct intervention in the already installed regulatory control system. This work presents a secondary frequency control scheme in an electric power system, through a hierarchical model predictive control (MPC). The regulatory level, corresponding to traditional primary and secondary control, will be maintained. An optimal additive signal is included, which is generated from a MPC algorithm, in order to optimize the behavior of the traditional secondary control system.
\end{abstract}

\section{Introduction}

The growing complexity of the power systems, due to the increased interconnection, the use of new technologies, and the need for operating the system based on economic indicators, has motivated the creation of some tools that enable the system to operate with a high degree of security and very close to the limits of stability conditions. The use of advanced control techniques has been an effective way of extending the limits of stability and improving the operation of EPS $[1,2]$. Model predictive control is one of such advanced control techniques and is presented below.

1.1. Model Predictive Control. Model predictive control is a control technique defined as a collection of past and present information to predict the future behavior of a system, through the explicit use of a process model. The generation of the control signal is made through the minimization of an objective function. Essentially, this control technique is based on the use of a finite slide horizon control, which involves the calculation of the control sequence for the whole horizon, but only the first control signal in the sequence is applied to the plant, and the process is repeated in the next sampling instant [3].

MPC is one of the few control techniques that allow the incorporation of variable constraints in the formulation. Furthermore, this control strategy is valid for a wide range of systems, linear and nonlinear, and has had a significant impact on the industry.

Guaranteeing the stability of the closed loop system is an essential aspect in the design of a controller. The stability analysis in the MPC is an aspect that has been evolving and now is considered as a mature field. The stability of the MPC controller is guaranteed due to the establishment of conditions (valid for most of the systems) [4]. These conditions are based on a formulation of the controller that includes cost terminal as well as terminal restrictions.

Determination of a more suitable hierarchical structure to control a power system is a very important task. The use of a single control system to operate the entire plant, or dividing the plant in a set of subsystems, and make the individual control of each one may represent a significant reduction in electricity costs if the best option is selected. In this paper we present the control techniques that gave better results in the problem. 
1.2. Applications of MPC in EPS. One of the most important applications of MPC in EPS is the voltage control, which can be defined as operational activities to keep the voltage within a permissible range into a specific sector, providing appropriate reactive power flow through the transmission system, at a particular time, with the objective of maximizing the active power flow [2]. Some recent MPC algorithm applications to control voltage can be cited, such as [5], where a predictive controller is implemented with a mixed logical dynamical model to control the voltage in a 12 bar network. Discrete control actions are load shedding, control transformer taps, and capacitor connection. A similar work is made in $[6,7]$, where a MPC to control the voltage of a 10 bar system is presented. The implementation includes a terminal region, which assures the stability of the controller, and the solution is obtained solving a linear programming problem. In [8], a MPC approach is used to prevent voltage instability in the long term. The MPC algorithm is based on a linearized steady state system model, derived from power flow equations. Simulations have been made in an EPS Nordic32 system. In [6], a method for optimal coordination of load shedding, capacitor switching, and taps changer is presented, in order to preserve the voltage stability of long-term. A dynamic model is used and a search tree is used as a method of solution for the MPC algorithm.

Another type of application of MPC in EPS is the control of power oscillations, which are variations on the three-phase power, due to the advance and retreat of the relative angles of voltage between generators, due to changes in the magnitude of the loads, faults, and other disturbances in the system [2]. We can cite works as [9], where a new control of generator excitation is proposed, to assure the stability of an EPS with multiple generators. The MPC algorithm is implemented using a DSP to achieve stability in real time with adequate speed. The simulations show that the interarea oscillation arising after a large disturbance in a connection line of two areas can be damped quickly. Besides, simulation shows that the stability of the MPC for multiple generators has a similar quality compared with the optimum excitation control using an automatic voltage regulator (AVR) of high gain in addition to a finely tuned power system stabilizer (PSS).

The harmonic control is an important issue in EPS too, due to the fact that it is considered a main indicator of the service quality. In [10], author argues that the increasing nonlinear loads generate problems due to the effects of the harmonic components of currents and voltages in an EPS, for example, cables overheating transformers and motors, excessive currents in the neutral resonance phenomena between the circuit elements $[11,12]$, and considering the capacitor banks for power factor correction. So, in general, the quality of the power supply is deteriorated by this distortion in voltages and currents [13-15]. Regarding the applications of MPC in harmonic control, we can mention the work in [16], which presents a modulation method based on the MPC and the sliding Fourier transform, including a low switching frequency and less distortion in lower order harmonics. The results are similar to the algorithm of selective harmonic mitigation [17]. However, the proposed technique is computed online and presents an improvement in the dynamic performances. The method can be applied to any converter topology with any number of levels in a simple manner. Results show that a large modulation index can be used to achieve excellent performance, even in the range of overmodulation.

Frequency control is defined as the ability of an EPS to keep the frequency constant after a disturbance, whose origin is a significant imbalance between generation and load. The literature related to the implementation of the MPC algorithm to control frequency of an EPS includes the area called automatic control of large scale systems. We can cite a decentralized frequency control presented in [18], using the MPC algorithm in a multiarea power. The MPC technique is designed such that the effect of the uncertainty due to variation of the parameters of the governor and turbine is reduced. Each local area controller is independently designed, so that the overall stability of the closed loop system is guaranteed. Model frequency response of EPS multizone is introduced, and the physical limitations of the governors and turbines have been considered. Note that in this decentralized controller the stability of the entire system is not guaranteed.

In [19] a MPC distributed control addressed the problem of cascading failures, which cause blackouts with high costs. A cascading failure can be thought of as an alternating sequence of equipment failures and violations of the dynamic constraints of the power system. The designed controller is described as a network of autonomous agents with quick response to reduce these sequences. Agents work in the elimination of violations before they can cause more interruptions. They make their decisions with a distributed MPC technique. Each agent has a set of models, specialized in its location in the network. The agent uses these models to predict what the other agents will do and how the network will respond. Then, each agent optimizes its decisions with respect to these predictions. A comparison study on the basic data-driven methods for process monitoring and fault diagnosis is presented in [20].

One remarkable work is the one in [21], which is the first establishing the distributed MPC systems stability. Based on noncooperative targets, this paper proposes a set of MPC subcontrollers, which transmits the information of the current state-entry trajectory to whole neighbors (MPC subcontrollers) with which it is interconnected and then competing subcontrollers have no knowledge of the other cost functions. From the game theory perspective, the balance of this strategy, if it exists, is called a noncooperative equilibrium or Nash equilibrium. The control objectives for each MPC subcontrol are often in conflict with the objectives the other MPC subcontrols have; that is, Nash equilibrium is usually suboptimal Pareto [22]. In addition, recent results on large scale system have been developed by [23].

Therefore, it is necessary to modify the objective function of each subcontroller, in order to provide a cooperation between them, which is achieved replacing in each subcontrol the noncooperative objective by one that measures the impact of control actions in the whole system. In this case, a convex combination of the objectives of the individual subsystems is used. With this modification, the best performance achievable by controllers is characterized by a Pareto optimal path, 


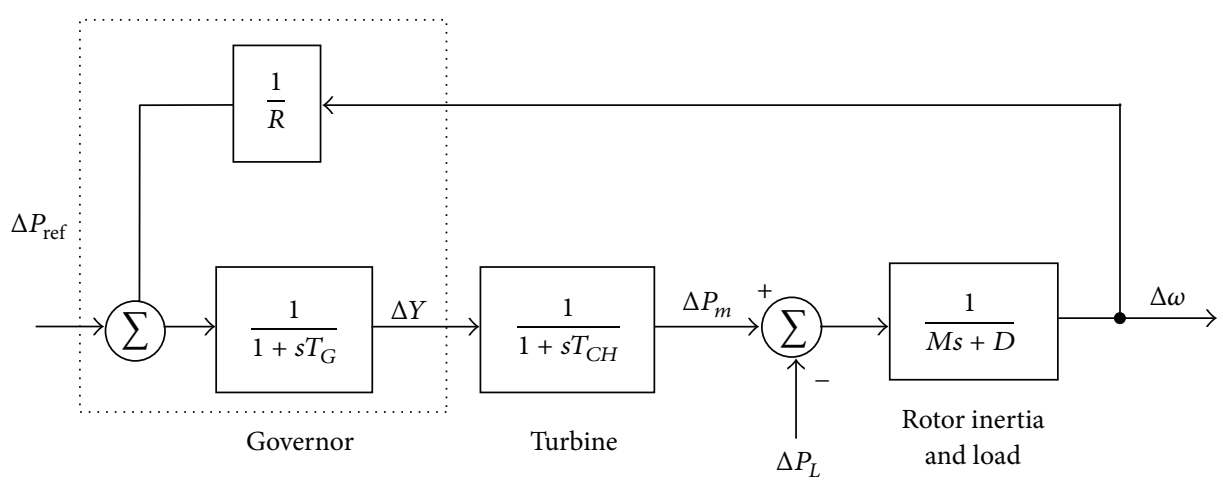

FIGURE 1: Primary control block diagram with governor, prime motor, rotating mass, and load.

which represents the optimal trade-off set between their goals and the goals of other systems with which there is conflict. Then, it can be proved that the mediator iterations generated by these MPC algorithms, based on cooperation, are feasible and the control law state feedback MPC algorithm based on these mediating iterations is asymptotically stable. As an application the authors achieve a secondary control of four areas in an EPS.

In [24], a comparison is made between a centralized, decentralized, and distributed MPC for an EPS. An important work has been developed by [25], where a distributed stable nonlinear dynamic control system is proposed, based on a set of MPC controllers that share only updated information of its neighbors (without predictions of the behavior of neighbors). Asymptotic stability in an equilibrium point (origin) in the distributed MPC controller is achieved with the use of socalled structured Lyapunov function control, applied to the respective MPC subcontrollers. Authors present an application in secondary control to a CIGRE EPS of seven machines, using a distributed predictive control. However, all the machines were used for this purpose, which is not reasonable due to economic and technical reasons.

In relation to renewable energy, there are two interesting works in [26], where an economic dispatch with intermittent sources is presented and describes an objective function that penalizes performance indices related to generation costs and environmental costs. The flexibility of MPC algorithm allows the use of constraints that limit the speed ramp for entry into service. The prediction, through a solar power plant model and turbine units model, allows dispatching units with slower speed ramp, with the respective economical savings (the generating units with faster speed ramp generally has higher operating costs). The work in [27] also includes an analysis of frequency stability of an EPS with intermittent sources (generic name that includes renewable sources).

\section{Classic Power System Control}

In this section we present classic power system control, which includes primary and secondary frequency control.

2.1. Primary Frequency Control. Primary control corresponds to an integral control action of each unit, due to its speed governors. This allows limiting frequency deviations from disturbance in the generation/load balance, in a few seconds of time response. However, the resulting frequency is not necessarily the nominal frequency, a result that is achieved with the secondary control, which is presented in Section 2.2. The block diagram of the governor, prime motor, rotating mass, and load is shown in Figure 1, where

$$
\begin{aligned}
& \Delta \omega=\omega_{r}-\omega_{0} \text { with } \omega_{r} \text { : real angular speed, and } \omega_{0} \text { : } \\
& \text { nominal angular speed; } \\
& D: \text { damping constant; } \\
& K \text { : constant PI control; } \\
& R \text { : statism; }
\end{aligned}
$$

$M: 2 H$ with $H$ the inertia constant;

$\Delta Y, \Delta P_{m}, \Delta P_{L}, \Delta P_{\text {ref }}$, and $\Delta \omega$ are the variations in position of the valve, mechanical power, load power, power reference, and frequency, respectively;

$T_{C H}, T_{G}=(1 / K R)$ are the time constants of the turbine and governor, respectively.

Considering a set of $n$-machines interconnected by transmission lines [25], the equations that represent the primary frequency control for the $i$ th machine are

$$
\begin{gathered}
\Delta \dot{\delta}_{i}=\Delta \omega_{i} \\
\Delta \dot{\omega}_{i}=\frac{1}{M_{i}}\left(\Delta P_{m_{i}}-D_{i} \Delta \omega_{i}-\Delta P_{L_{i}}-\sum_{\left\{j \mid\left(\zeta_{i}, \varsigma_{j}\right) \in \bar{\varepsilon}\right\}} \Delta P_{\mathrm{tie}_{i, j}}\right) \\
\Delta \dot{P}_{m_{i}}=\frac{1}{T_{C H}}\left(\Delta Y_{i}-\Delta P_{m_{i}}\right), \\
\Delta \dot{Y}_{i}=\frac{1}{T_{G_{i}}}\left(\Delta P_{\text {ref }_{i}}-\Delta Y_{i}-\frac{1}{R_{i}} \Delta \omega_{i}\right) \text { con } T_{G_{i}}=\frac{1}{K_{i} R_{i}} .
\end{gathered}
$$

We assume that $P_{\mathrm{tie}_{i, j}}$ is the transmitted power by the transmission line $i j$, where $\left(\varsigma_{i}, \varsigma_{j}\right)$ represents the transmission line between node $\varsigma_{i}$ and node $\varsigma_{j}$, belonging to the set of arcs $\bar{\varepsilon}$ and coupling (5) relating the angles of a load $\delta_{j}, j \in\{j \mid$ 
$\left.\varsigma_{j} \in S_{\text {Load }}\right\}$, with the angles and frequencies of the generators $\delta_{i}, \omega_{i}, i \in\left\{i \mid \varsigma_{i} \in S_{\text {Generator }}\right\}$. Consider

$$
\begin{gathered}
{\left[\begin{array}{c}
M_{1} \Delta \dot{\omega}_{1} \\
\vdots \\
M_{N g} \Delta \dot{\omega}_{N g} \\
\hline 0 \\
\vdots \\
0
\end{array}\right]} \\
=\left[\begin{array}{c}
\Delta P_{m_{1}}-D_{1} \Delta \omega_{1}-\Delta P_{L_{1}} \\
\vdots \\
\Delta P_{m_{N g}}-D_{N g} \Delta \omega_{N g}-\Delta P_{L_{N g}} \\
\hline-\Delta P_{L_{N g+1}} \\
\vdots \\
-\Delta P_{L_{\text {nod }}} \\
\\
\delta_{1} \\
\vdots \\
\delta_{N g}
\end{array}\right] \\
-\left[\begin{array}{c|c}
B_{11} & B_{12} \\
\hline B_{21} & B_{22}
\end{array}\right]\left[\begin{array}{c}
\delta_{N g+1} \\
\vdots \\
\delta_{\text {nod }}
\end{array}\right]
\end{gathered}
$$

where the admittance matrix

$$
B=\left[\begin{array}{ll}
B_{11} & B_{12} \\
B_{21} & B_{22}
\end{array}\right]
$$

is suitably divided into submatrices $B_{11} \in M_{N g \times N g}$, $B_{12} \in M_{N g \times(\text { nod-Ng) }}, B_{21} \in M_{(\operatorname{nod}-N g) \times N g}$, and $B_{22} \in$ $M_{(\text {nod }-N g) \times(\text { nod }-N g)}$, with $N g$ generators and nod nodes.

Eliminating the angles that represent the bar that does not have generators $\delta_{N g+1}, \ldots, \delta_{\text {nod }}[25]$,

$$
\left[\begin{array}{c}
M_{1} \Delta \dot{\omega}_{1} \\
\vdots \\
M_{N g} \Delta \dot{\omega}_{N g}
\end{array}\right]=\left[\begin{array}{c}
\Delta P_{m_{1}}-D_{1} \Delta \omega_{1} \\
\vdots \\
\Delta P_{m_{N g}}-D_{N g} \Delta \omega_{N g}
\end{array}\right]
$$

$$
-\Gamma\left[\begin{array}{c}
\delta_{1} \\
\vdots \\
\delta_{N g}
\end{array}\right]+\Upsilon\left[\begin{array}{c}
\Delta P_{L_{1}} \\
\vdots \\
\Delta P_{L_{\mathrm{nod}}}
\end{array}\right],
$$

where

$$
\begin{gathered}
\Gamma:=\left(B_{11}-B_{11} B_{22}^{-1} B_{21}\right), \\
\Upsilon:=\left[\begin{array}{ll}
-I_{N g} & B_{12} B_{22}^{-1}
\end{array}\right] .
\end{gathered}
$$

Then, to represent the continuous dynamic model of EPS with $\mathrm{Ng}$ generators and nod nodes we define the following matrices:

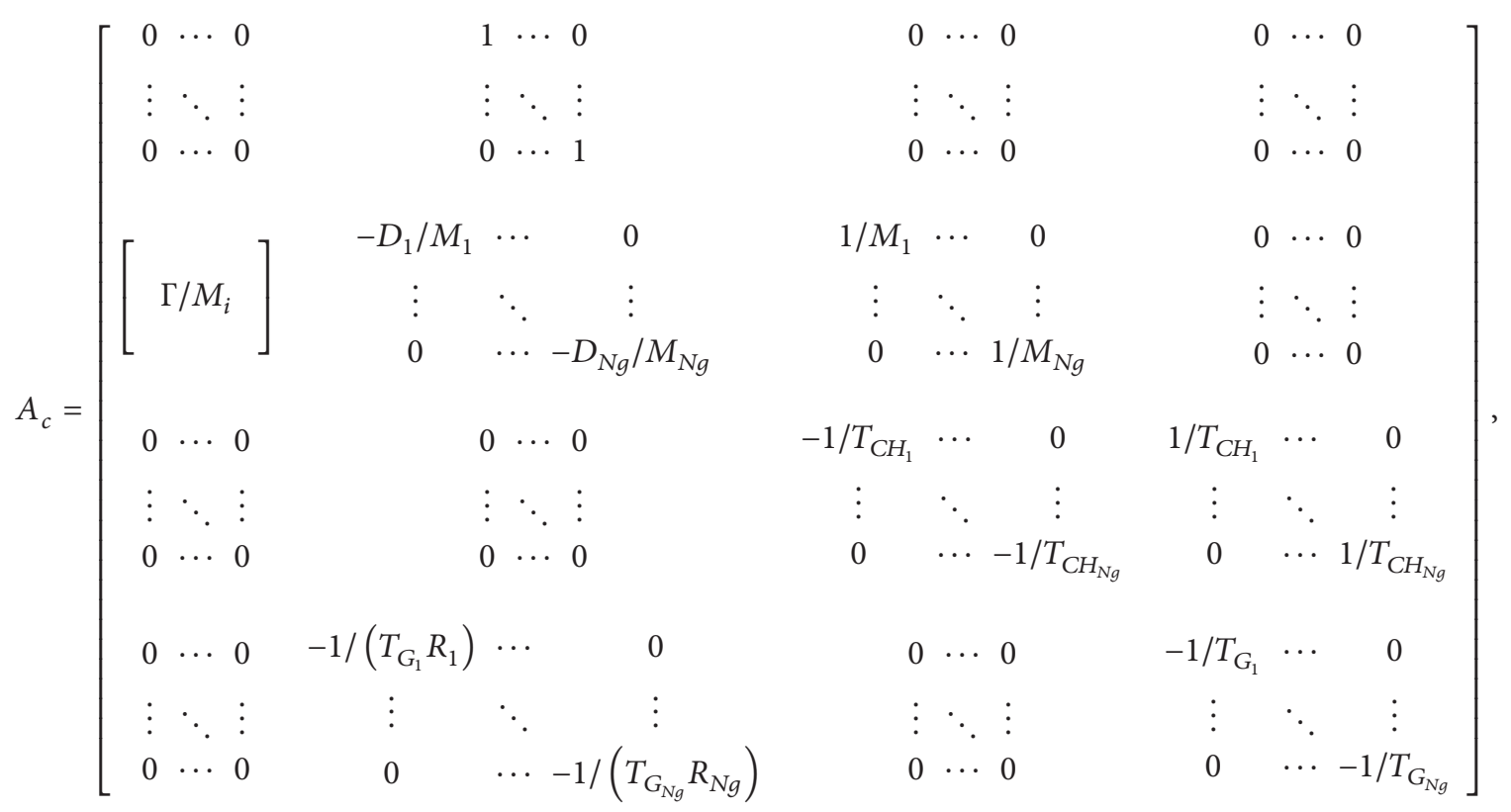




$$
\begin{aligned}
& \text { where }\left[\Gamma / M_{i}\right] \equiv\left[\begin{array}{ccc}
\Gamma_{11} / M_{1} & \cdots & \Gamma_{1 n} / M_{1} \\
\vdots & & \vdots \\
\Gamma_{n 1} / M_{n} & \cdots & \Gamma_{n n} / M_{n}
\end{array}\right] \text {, and }
\end{aligned}
$$

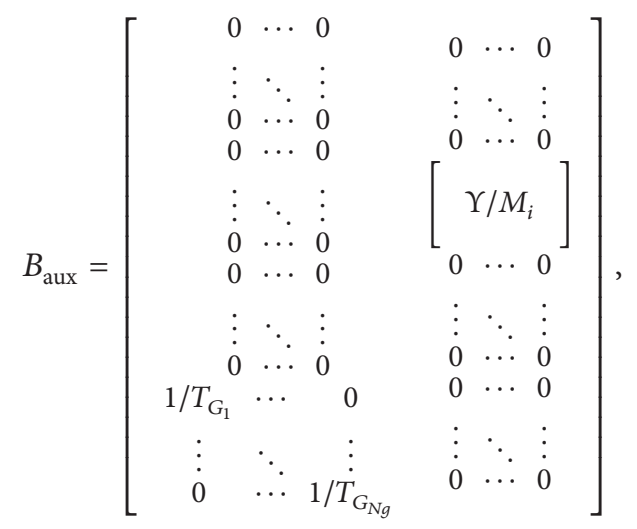

with $\left[\Upsilon / M_{i}\right] \equiv\left[\begin{array}{ccc}\Upsilon_{11} / M_{1} & \cdots & \Upsilon_{1 n} / M_{1} \\ \vdots & & \vdots \\ \Upsilon_{n 1} / M_{n} & \cdots & \Upsilon_{n n} / M_{n}\end{array}\right]$.

Then, the continuous dynamic model can be represented as

$$
\dot{x}=A_{c} x+B_{\text {aux }} u,
$$

with

$$
\begin{aligned}
& x= \\
& {\left[\begin{array}{cccccccccccc}
\Delta \delta_{1} & \cdots & \Delta \delta_{N g} & \Delta \omega_{1} & \cdots & \Delta \omega_{N g} & \Delta P_{M_{1}} & \cdots & \Delta P_{M_{N g}} & \Delta Y_{1} & \cdots & \Delta Y_{N g}
\end{array}\right]^{\prime},} \\
& u=\left[\begin{array}{llllllll}
\Delta P_{\mathrm{ref}_{1}} & \cdots & \Delta P_{\mathrm{ref}_{N g}} & \Delta P_{L_{1}} & \cdots & \Delta P_{L_{\text {nod }}}
\end{array}\right]^{\prime} .
\end{aligned}
$$

2.2. Secondary Frequency Control. The role of secondary frequency control is to keep or restore the system frequency to its nominal value, the balance generation/load within a control area, and scheduled power exchanges with neighboring areas of control. This control is addressed modifying the set-point of active power units assigned to secondary control, which belong to the control area where the imbalance occurs.

The secondary frequency control can be manually accomplished by instructions of the plant operators or automatically by the automatic generation control. Unlike the primary control, its action is slow and coordinated, taking into account characteristics of the units, such as its speed of response (however for the current job this will not be considered). This control covers a time from the end of the action of the primary control to several minutes and should not interfere with the action of the secondary control.

Now, area error control (ACE) value necessary for the secondary frequency power control is developed. In Figure 2, the connection between neighbors areas $i$ and $i+1$ and also incoming and outgoing power flow are shown. Sets $\Omega_{i}$ and $\Psi_{i}$ are defined as follows:

$$
\begin{aligned}
& \Omega_{i} \text { : set of neighbors areas to } i \text { which inject power; } \\
& \Psi_{i} \text { : set of neighbors areas to } i \text { which demand power. }
\end{aligned}
$$

For each area, the steady state dynamic equation depends on the variation of mechanical power, the incoming net flow,

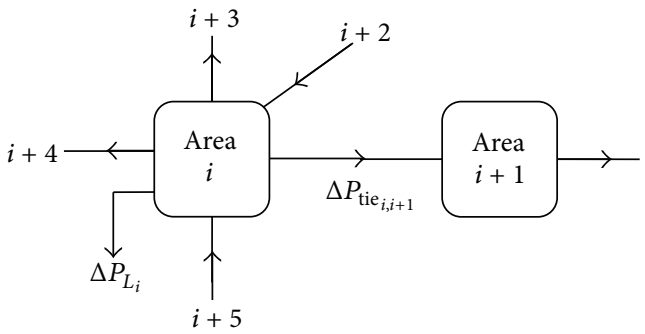

FIGURE 2: Connection between areas.

and the outgoing net flow. Assuming that there is a load variation $\Delta P_{L_{i}}$ in the $i$ th area

$$
\Delta P_{\text {mech }_{1}}+\sum_{j \in \Omega_{1}} \Delta P_{\mathrm{tie}_{1, j}}-\sum_{k \in \Psi_{1}} \Delta P_{\mathrm{tie}_{1, k}}=\Delta \omega D_{1},
$$

$$
\Delta P_{\text {mech }_{i}}+\sum_{j \in \Omega_{i}} \Delta P_{\mathrm{tie}_{i, j}}-\sum_{k \in \Psi_{i}} \Delta P_{\mathrm{tie}_{i, k}}-\Delta P_{L_{i}}=\Delta \omega D_{i},
$$

$$
\Delta P_{\text {mech }_{n}}+\sum_{j \in \Omega_{n}} \Delta P_{\text {tie }_{n, j}}-\sum_{k \in \Psi_{n}} \Delta P_{\text {tie }_{n, k}}=\Delta \omega D_{n}
$$

then for each area frequency power variation ratio is

$$
\Delta P_{\text {mech }_{i}}=-\frac{\Delta \omega_{i}}{R_{i}}
$$

where $R_{i}$ is equivalent statism of $i$ th area; also $\beta_{i}=\left(1 / R_{i}\right)+D_{i}$. Summing the equations in (13), then

$$
\sum_{i=1}^{n} \Delta P_{\text {mech }_{i}}-\Delta P_{L_{i}}=\Delta \omega \sum_{i=1}^{n} D_{i}
$$

replacing $\Delta P_{\text {mech }_{i}}$ and $\beta_{i}=\left(1 / R_{i}\right)+D_{i}$, implies $-\Delta P_{L_{i}}=$ $\Delta \omega \sum_{i=1}^{n} \beta_{i}$. Then we come to the traditional result of the frequency variation for the complete system

$$
\Delta \omega=-\frac{\Delta P_{L_{i}}}{\sum_{i=1}^{n} \beta_{i}} .
$$



(13)

Now, using the power frequency variation ratio (14) in

$$
\begin{aligned}
& \sum_{j \in \Omega_{1}} \Delta P_{\mathrm{tie}_{1, j}}-\sum_{k \in \Psi_{1}} \Delta P_{\mathrm{tie}_{1, k}}=\Delta \omega \beta_{1} \\
& \sum_{j \in \Omega_{i}} \Delta P_{\mathrm{tie}_{i, j}}-\sum_{k \in \Psi_{i}} \Delta P_{\mathrm{tie}_{i, k}}-\Delta P_{L_{i}}=\Delta \omega \beta_{i} \\
& \sum_{j \in \Omega_{n}} \Delta P_{\mathrm{tie}_{n, j}}-\sum_{k \in \Psi_{n}} \Delta P_{\mathrm{tie}_{n, k}}=\Delta \omega \beta_{n} .
\end{aligned}
$$

From equation of the $i$ th area, (16), we see that the power variation introduced in this area $-\Delta P_{L_{i}}=-\sum_{j \in \Omega_{i}} \Delta P_{\text {tie }_{i, j}}+$ $\sum_{k \in \Psi_{i}} \Delta P_{\text {tie }_{i, k}}+\Delta \omega \beta_{i}$, which provides a motivation to define the error area $\mathrm{AEC}_{i}$, should be fed back to the reference power of the secondary controller frequency of $i$ th area

$$
\mathrm{ACE}_{i}=-\sum_{j \in \Omega_{i}} \Delta P_{\mathrm{tie}_{i, j}}+\sum_{k \in \Psi_{i}} \Delta P_{\mathrm{tie}_{i, k}}+\Delta \omega \beta_{i} .
$$

Now, as load variation $\Delta P_{L_{i}}$ occurs in the $i$ th area, summing all equations in (5) except the $i$ th, where load variation occurred $-\sum_{j \in \Omega_{i}} \Delta P_{\text {tie }_{i, j}}+\sum_{k \in \Psi_{i}} \Delta P_{\text {tie }_{i, k}}=\Delta \omega \sum_{k=1, k \neq i} \beta_{k}$, and using (16)

$$
-\sum_{j \in \Omega_{i}} \Delta P_{\mathrm{tie}_{i, j}}+\sum_{k \in \Psi_{i}} \Delta P_{\mathrm{tie}_{i, k}}=-\Delta P_{L_{i}} \frac{\sum_{k=1, k \neq i}^{n} \beta_{k}}{\sum_{i=1}^{n} \beta_{i}} .
$$

Therefore in (6) using (7) and (4)

$$
\begin{aligned}
\mathrm{ACE}_{i} & =-\Delta P_{L_{i}} \frac{\sum_{k=1, k \neq i}^{n} \beta_{k}}{\sum_{i=1}^{n} \beta_{i}}-\Delta P_{L_{i}} \frac{\beta_{i}}{\sum_{i=1}^{n} \beta_{i}} \\
& =-\Delta P_{L_{i}} \frac{\sum_{k=1}^{n} \beta_{k}}{\sum_{i=1}^{n} \beta_{i}}=-\Delta P_{L_{i}} .
\end{aligned}
$$

Now, summing all equations in (17), except the equation of any area different from the $i$ th area where there was load variation $\Delta P_{L_{i}}$,

$$
-\sum_{j \in \Omega_{i}} \Delta P_{\mathrm{tie}_{i, j}}+\sum_{k \in \Psi_{i}} \Delta P_{\mathrm{tie}_{i, k}}-\Delta P_{L_{i}}=\Delta \omega \sum_{\substack{k=1 \\ k \neq i}}^{n} \beta_{k} .
$$

Using (4)

$$
\begin{gathered}
-\sum_{j \in \Omega_{i}} \Delta P_{\mathrm{tie}_{i, j}}+\sum_{k \in \Psi_{i}} \Delta P_{\mathrm{tie}_{i, k}}+\Delta \omega \sum_{i=1}^{n} \beta_{i}=\Delta \omega \sum_{\substack{k=1 \\
k \neq i}}^{n} \beta_{k}, \\
-\sum_{j \in \Omega_{i}} \Delta P_{\mathrm{tie}_{i, j}}+\sum_{k \in \Psi_{i}} \Delta P_{\mathrm{tie}_{i, k}}=-\Delta \omega \beta_{i} .
\end{gathered}
$$

Therefore

$$
\mathrm{ACE}_{i}=-\Delta \omega \beta_{i}+\Delta \omega \beta_{i}=0
$$

Summarizing,

$$
\begin{gathered}
\mathrm{ACE}_{1}=0 \\
\vdots \\
\mathrm{ACE}_{i}=-\Delta P_{i} \\
\vdots \\
\mathrm{ACE}_{n}=0 .
\end{gathered}
$$

This shows that the selection of error area, as in (18), guarantees that the area where the power variation occurred provides the required power, and all this is in steady state.

Summarizing, given an interconnection of $N$ control areas, suppose a disturbance load $\Delta P_{i}$ appears in the $i$ th area. During the transient period, the dynamic phenomena involve generation of different frequencies in each area and deviations in the flow of power between them, calculating each area with its own area control error $\mathrm{ACE}_{i}(18)$. Then we defined the error in the net power exchanged $\Delta P_{\text {tie }_{i}}$ from all neighboring areas to the area $i$ :

$$
\Delta P_{\mathrm{tie}_{i}}=-\sum_{j \in \Omega_{i}} \Delta P_{\mathrm{tie}_{i, j}}+\sum_{k \in \Psi_{i}} \Delta P_{\mathrm{tie}_{i, k}},
$$

and then area control error can be written as

$$
\mathrm{ACE}_{i}=\Delta P_{\mathrm{tie}_{i}}+\beta_{i} \Delta \omega
$$
by

Finally, the new reference $P_{\text {ref }_{i}}$ of generator $i$ is expressed

$$
P_{\mathrm{ref}_{i}}=-K_{i} \int_{0}^{\tau} \mathrm{ACE}_{i} d t
$$

\section{Design of Hierarchical MPC for EPS}

In this section we present the design of a frequency hierarchical MPC for EPS, including restrictions and optimization problem.

3.1. Problem Statement. The control strategy proposed in this paper is based on a hierarchical supervisor level, which determines the optimal set-point for a given regulatory system. The supervisor level dynamically optimizes a general objective function including equality and inequality constraints. Then, the described problem can be solved analytically with the predictive control theory and can be solved by numerical algorithms when working with restrictions [3].

Figure 3 shows how the hierarchical supervisor level delivery set-point $r$ is based on the optimization of the objective function $J$, the trajectory of an external reference $W$, controlled variable $y$, and manipulated variable $u$. The process is influenced by a nonmeasurable disturbance $p$. 


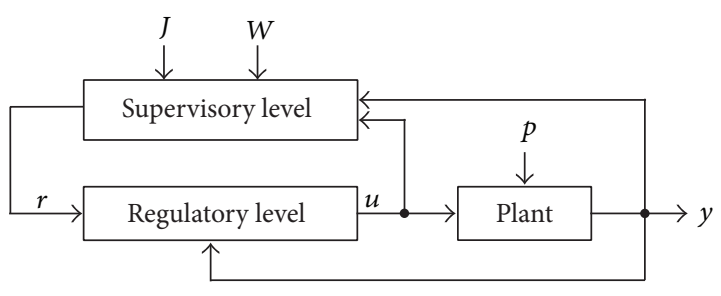

FIGURE 3: Supervisory control diagram.

3.2. Application of Secondary Control of EPS. The implementation of MPC control for the hierarchical secondary control of an EPS is presented in Figure 4. The figure presents an area, bounded by a dotted line, which represents the linear EPS model including a primary frequency control. Besides, in the traditional secondary control, we have included an additive power signal $P_{\mathrm{MPC}}$, which provides an optimum correction to signal of ACE. The correction is optimal because, in the optimization MPC problem, the objective function strongly penalizes the frequency variation and includes restrictions, which represent plant model and traditional secondary controller model.

3.3. Restrictions of EPS Model for the Optimization Problem. The corresponding restrictions for building optimization problem for the MPC algorithm are presented below. The involved variables must behave according to the dynamic model of the EPS. Then discretizing the model obtained in (11) we obtain

$$
A\left(T_{s}\right)=e^{A_{c} T_{s}}, \quad B_{\mathrm{aux}}\left(T_{s}\right)=\left(e^{A_{c} T_{s}}-I\right)(A c)^{-1} B_{\mathrm{aux}_{c}}
$$

with

$$
\begin{aligned}
& T_{s}=1[\mathrm{~s}], \\
& B_{\text {aux }}=\left[\begin{array}{ll}
B & B_{L}
\end{array}\right] \text {, } \\
& u(k)=\left[\begin{array}{lll}
\Delta P_{\mathrm{ref}_{1}}(k) & \cdots & \Delta P_{\mathrm{ref}_{N g}}(k)
\end{array}\right]^{\prime}, \\
& P_{L}(k)=\left[\Delta P_{L_{1}}(k) \cdots \Delta P_{L_{\text {nod }}}(k)\right]^{\prime}, \\
& x(k)=\left[\begin{array}{lllllllllllll}
\Delta \delta_{1}(k) & \cdots & \Delta \delta_{N g}(k) & \Delta \omega_{1}(k) & \cdots & \Delta \omega_{N g}(k) & \Delta P_{M_{1}}(k) & \cdots & \Delta P_{M_{N g}}(k) & \Delta Y_{1}(k) & \cdots & \Delta Y_{N g}(k)
\end{array}\right]^{\prime} .
\end{aligned}
$$

Hence, we have the following discrete model:

$$
x(k+1)=A x(k)+B u(k)+B_{L} P_{L}(k) .
$$

The group of restrictions to $N$ steps of EPS model used for the corresponding MPC optimization problem is

$$
\begin{gathered}
-B_{L} P_{L}(k)-A x(k)-B u(k)=-x(k+1) \\
-B_{L} P_{L}(k+1)=-x(k+2)+A x(k+1)+B u(k+1) \\
\vdots \\
-B_{L} P_{L}(k+N-1) \\
=-x(k+N)+A x(k+N-1)+B u(k+N-1),
\end{gathered}
$$

where $P_{L}$ model is obtained from a lineal model.

3.4. Constraints That Relate Angles. It is necessary to include the relationship between the angles of the bars without generators $\delta_{n g}$ with the angles of the bars containing the generators $\delta_{g}$.

Given the admittance matrix $\left[\begin{array}{ll}B_{11} & B_{12} \\ B_{21} & B_{22}\end{array}\right]$,

$$
\begin{gathered}
B_{11} \in M_{N g \times N g}, \quad B_{12} \in M_{N g \times(\operatorname{nod}-N g)}, \\
B_{21} \in M_{(\operatorname{nod}-N g) \times N g}, \quad B_{22} \in M_{(\operatorname{nod}-N g) \times(\operatorname{nod}-N g)} .
\end{gathered}
$$

The group of restrictions to $N$ steps that shows that relationship between the angles can be deduced from (5) as follows:

$$
\begin{gathered}
-I_{n g} P_{L n g}(k)-B_{21} \delta_{g}(k)=-B_{22} \delta_{n g}(k) \\
0=-I_{n g} P_{L n g}(k+1)-B_{21} \delta_{g}(k+1)-B_{22} \delta_{n g}(k+1) \\
\vdots \\
0=-I_{n g} P_{L n g}(k+N)-B_{21} \delta_{g}(k+N)-B_{22} \delta_{n g}(k+N),
\end{gathered}
$$

with

$$
\begin{gathered}
I_{n g} \in M_{(\text {nod }-N g) \times(\operatorname{nod}-N g)}, \\
P_{L n g}(k)=\left[\begin{array}{llll}
\Delta P_{L_{N g+1}}(k) & \cdots & \Delta P_{L_{\text {nod }}}(k)
\end{array}\right]^{\prime}, \\
\delta_{g}(k+N)=\left[\begin{array}{llll}
\Delta \delta_{1}(k) & \cdots & \Delta \delta_{N g}(k)
\end{array}\right]^{\prime}, \\
\delta_{n g}(k+N)=\left[\begin{array}{llll}
\Delta \delta_{N g+1}(k) & \cdots & \Delta \delta_{\text {nod }}(k)
\end{array}\right]^{\prime} .
\end{gathered}
$$

3.5. Secondary Control Equations. The constraints that represent the secondary frequency control can be deduced applying the Laplace transform to the equation of secondary 


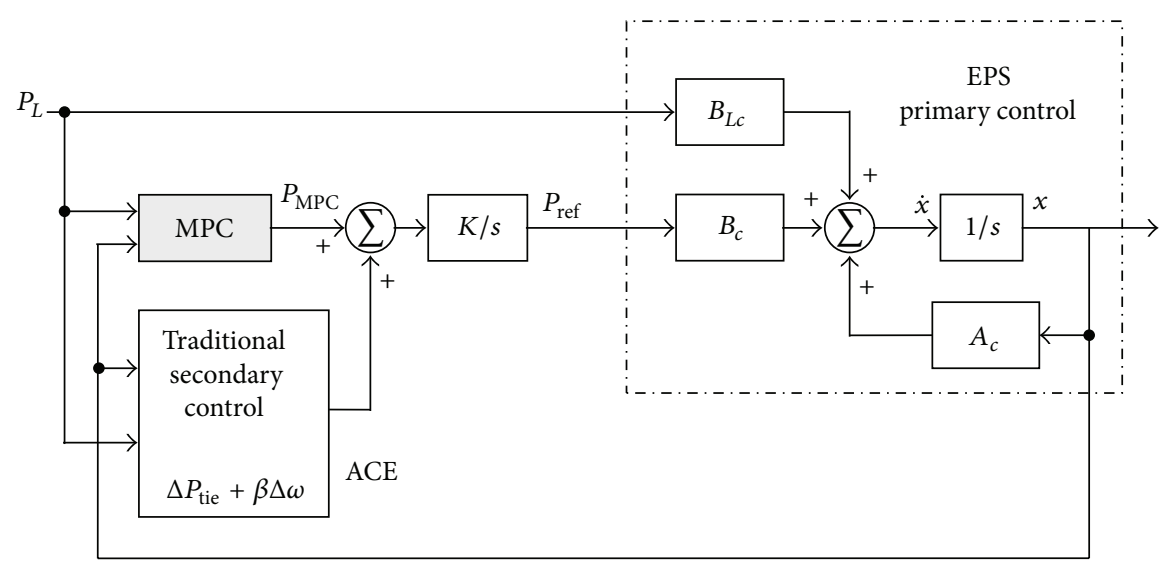

FiguRE 4: Hierarchical MPC control diagram.

control in (26) including an additive signal power $P_{\mathrm{MPC}_{i}}$ to area control error $\mathrm{ACE}_{i}$ :

$$
P_{\mathrm{ref}_{i}}=-K_{i} \frac{\mathrm{ACE}_{i}+P_{\mathrm{MPC}_{i}}}{s}, \quad i=1, \ldots, G 2,
$$

with G2 number generators with secondary control.

Using the Tustin triangular approximation, $(1 / s)=$ $\left(T_{s}(z+1)\right) /(2(z-1))$, where $T_{s}$ is the sampling time, we obtain

$$
\begin{aligned}
\frac{P_{\mathrm{ref}_{i}}(k)}{\mathrm{ACE}_{i}(k)+P_{\mathrm{MPC}_{i}}(k)} \\
=-K_{i} \frac{T_{s}\left(1+z^{-1}\right)}{2\left(1-z^{-1}\right)} P_{\mathrm{ref}_{i}}(k+1)-P_{\mathrm{ref}_{i}}(k) \\
=-\frac{T_{s} K_{i}}{2} \mathrm{ACE}_{i}(k+1)-\frac{T_{s} K_{i}}{2} P_{\mathrm{MPC}_{i}}(k+1) \\
-\frac{T_{s} K_{i}}{2} \mathrm{ACE}_{i}(k)-\frac{T_{s} K_{i}}{2} P_{\mathrm{MPC}_{i}}(k) .
\end{aligned}
$$

Then, we can generate the corresponding secondary control restrictions for $N$ steps. Finally the complete optimization problem is presented.
3.6. Formulation of MPC Optimization. The formulation of the optimization problem for the frequency MPC of the EPS is as follows.

Given in (30), the discrete model of the EPS is

$$
x(k+1)=A x(k)+B u(k)+B_{L} P_{L}(k) .
$$

The frequency control of the EPS by the MPC algorithm involves solving optimization problem (38) to find the optimal set $\left\{u^{*}(k), \ldots, u^{*}\left(k+N_{p}-1\right)\right\}$ of control actions to $N_{p}$ steps and apply as control action the single signal $u^{*}(k)$ :

$$
\min _{\left\{u(k), \ldots, u\left(k+N_{p}-1\right)\right\}} J=X(k)^{\prime} Q X(k)+U(k)^{\prime} R U(k),
$$

subject to the following:

constraints EPS model, (18),

constraints of relationship between $\delta_{n g}$ with $\delta_{g},(19)$, constraints of secondary control, (22), constraints of variables,

where

$$
\begin{aligned}
& Q \in M_{4 N_{p} N_{g} \times 4 N_{p} N_{g}} \text {, weights diagonal matrix; } \\
& R \in M_{N_{p} N_{g} \times N_{p} N_{g}} \text {, weights diagonal matrix. }
\end{aligned}
$$

Consider

$$
\begin{aligned}
& X(k)=\left[\begin{array}{lll}
x(k)^{\prime} & \cdots & x\left(k+N_{p}\right)^{\prime}
\end{array}\right] \in \mathbb{R}^{4 N_{p} N_{g}}, \\
& U(k)=\left[\begin{array}{lll}
u(k)^{\prime} & \cdots & u\left(k+N_{p}\right)^{\prime}
\end{array}\right] \in \mathbb{R}^{N_{p} N_{g}}, \\
& x(k)=\left[\begin{array}{lllllllllllll}
\Delta \delta_{1}(k) & \cdots & \Delta \delta_{N g}(k) & \Delta \omega_{1}(k) & \cdots & \Delta \omega_{N g}(k) & \Delta P_{M_{1}}(k) & \cdots & \Delta P_{M_{N g}}(k) & \Delta Y_{1}(k) & \cdots & \Delta Y_{N g}(k)
\end{array}\right]^{\prime}, \\
& u(k)=\left[\begin{array}{llll}
P_{\mathrm{MPC}_{1}}(k) & \cdots & P_{\mathrm{MPC}_{\mathrm{G} 2}}(k)
\end{array}\right]^{\prime}, \\
& u^{*}(k)=\left[\begin{array}{llll}
P_{\mathrm{MPC}_{1}}^{*}(k) & \cdots & P_{\mathrm{MPC}_{\mathrm{G} 2}}^{*}(k)
\end{array}\right]^{\prime} \text {. }
\end{aligned}
$$




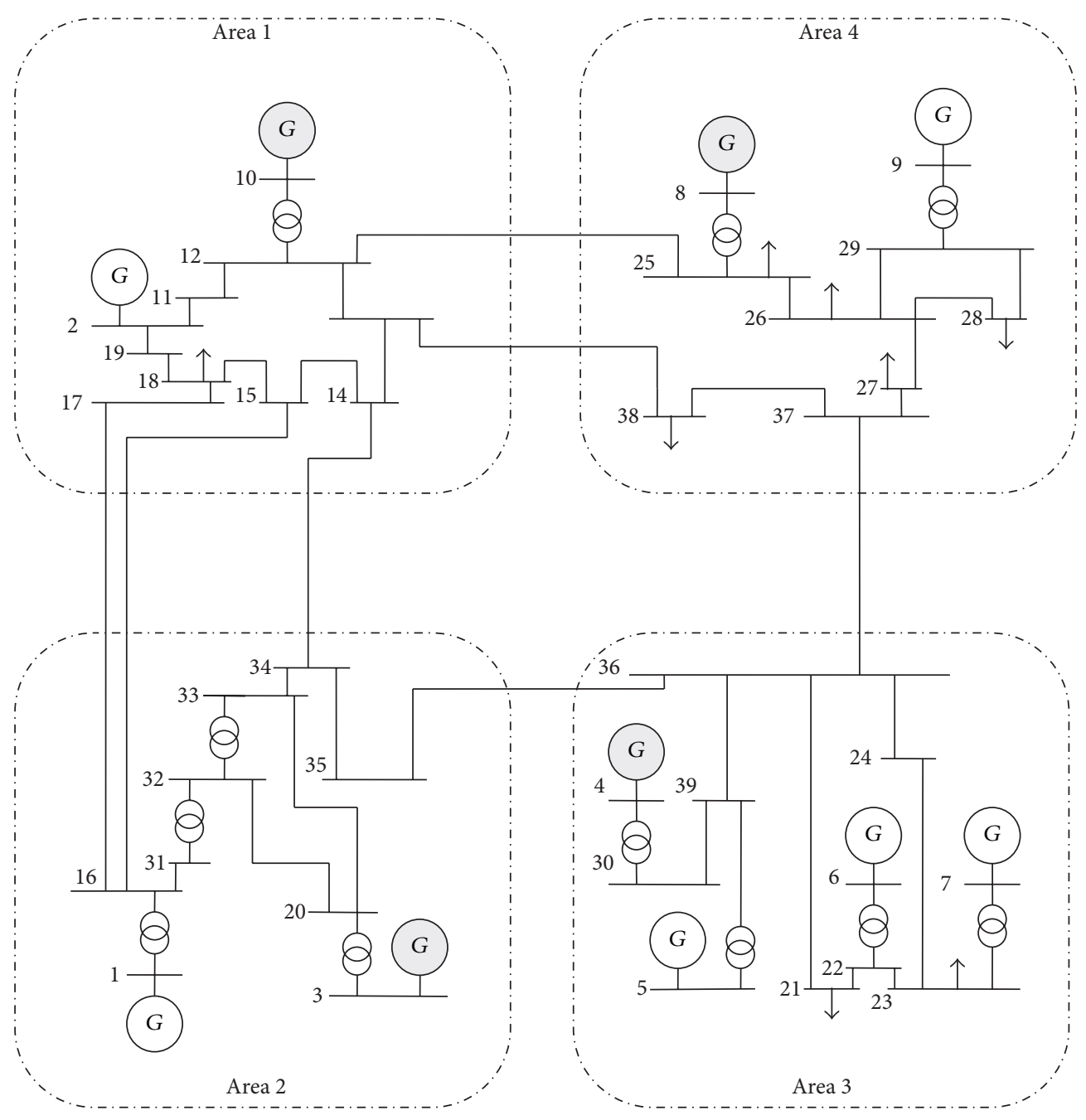

Figure 5: EPS IEEE-39 diagram.

TABLE 1: Secondary control generator.

\begin{tabular}{lc}
\hline Area & Generator \\
\hline 1 & 10 \\
2 & 3 \\
3 & 4 \\
4 & 8 \\
\hline
\end{tabular}

\section{Practical Application}

As a practical application, a secondary MPC control to EPS IEEE-39 of 39 bars and 10 generators is developed. The system is divided into four interconnected areas. In each area, a generator is designated for the secondary control (see Table 1). The unifilar diagram of the EPS is shown in Figure 5. The characteristics of the test system, generators, lines, and transformers impedances are presented in [1].
4.1. Results. Because the objective of the MPC algorithm is a quadratic form function and constraints are linear equations, the solution of the optimization problem presented is obtained by quadratic programming. In particular, prediction was performed at 10 steps with computation time of 0.75 [s] per iteration (using the Matlab program Quadprog), time less than the sampling interval of $1[\mathrm{~s}]$, which makes feasible the real-time control.

Figure 6 shows the response of the secondary frequency MPC controller to an increased load of $10 \%$ on bar 18 in area 1 of EPS IEEE-39. It can be seen, in Figure 6(a), that the MPC controller, within a reasonable time of 50 [s], achieves the convergence to zero of the frequency variations of all the generators.

In Figure 6(b), it can be seen that the generators making the secondary control (generators 3, 4, 5, and 11) are the ones that provide the required power (blue line) for satisfaction load variation, whereas the other generators only contribute in the initial stage. In Figures 6(c) and 6(d), the contribution 


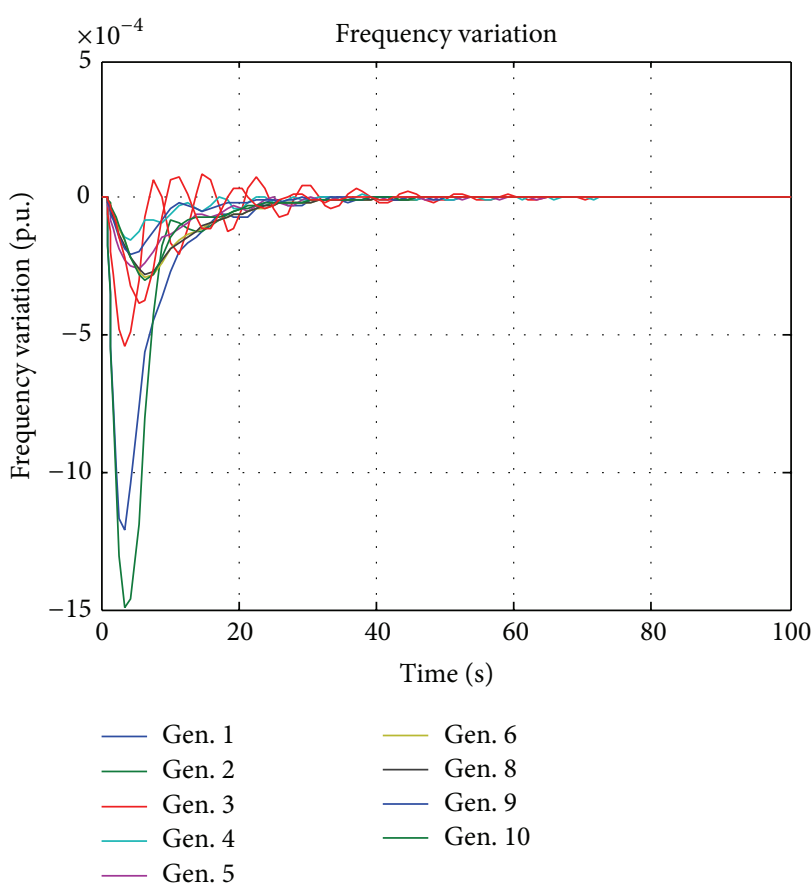

(a)

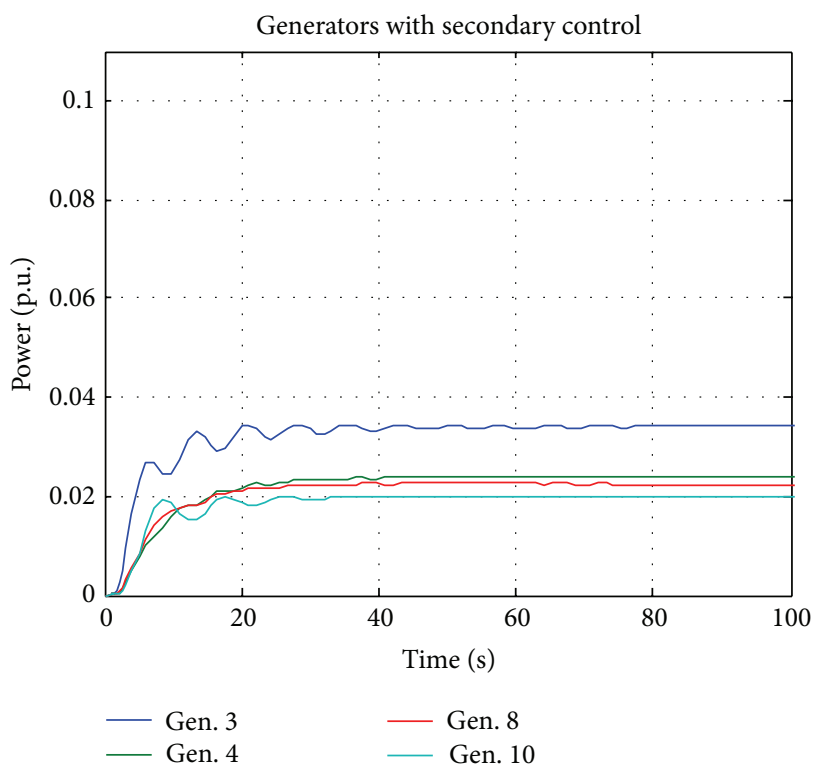

(c)

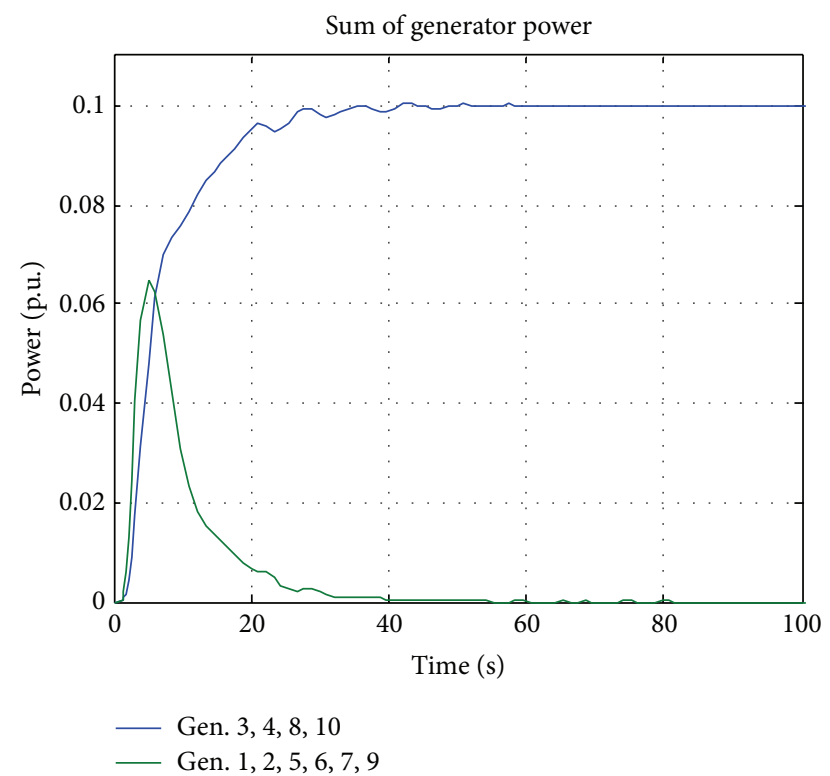

(b)

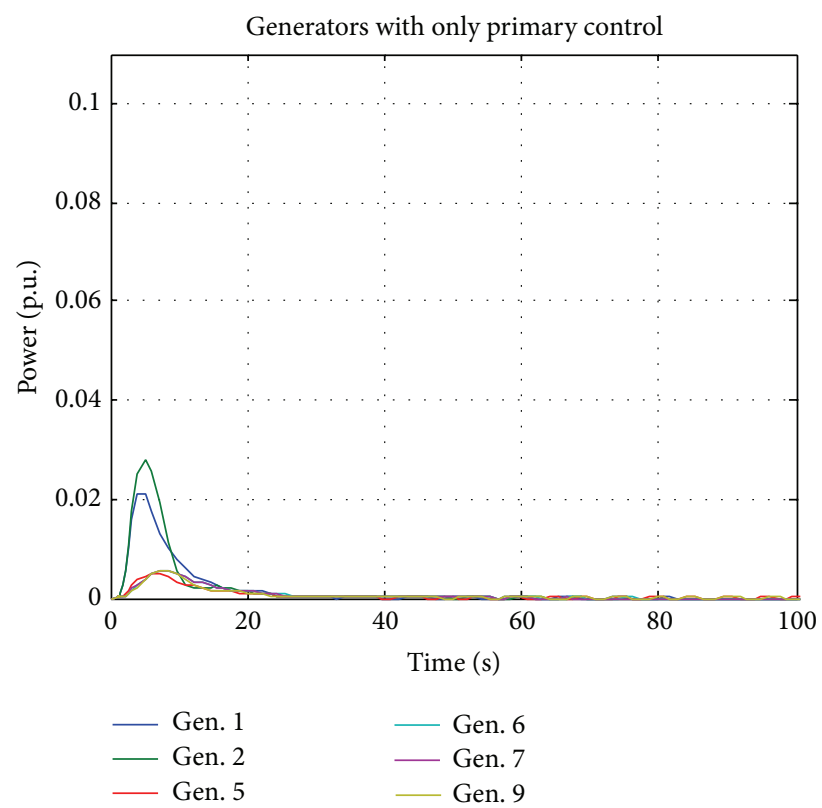

(d)

FIGURE 6: Response of the secondary frequency MPC controller.

of each generator separately can be seen, the ones that make secondary control (see Figure 6(c)) and the remaining generators (see Figure 6(d)). The greatest contribution of the power is given precisely by generator 3 , as can be seen in Figure 6(c), which made the secondary control in area 1 , where the load variation takes place.

Similar results (Figure 7) were achieved when simulating over 1500 [s], where the EPS was subjected to a series of power variations (Figure 7), not exceeding 15\%, in bar 18 in area 1.
Then $P_{L}$ model to (31) is obtained from a lineal model series of Figure 7.

Note that this work is in a framework of small signal analysis, implying linear models as an approximation of the system. However, by the necessity of using a predictive model of the system and for a more realistic treatment of the problem, we consider in future models nonlinear load variation, for example, Takagi and Sugeno or neural models; details of this type of model can be seen in [28-32]. 


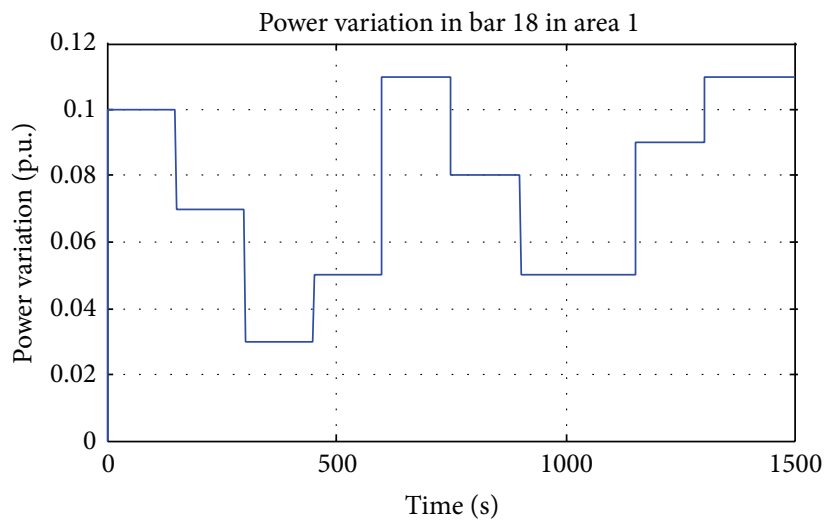

Figure 7: Power variations series $\left(P_{L}\right)$.

In Figure 8(a) we can see how the frequency variation converges to zero for different power variations. Due to similarity of results between control techniques, classical and MPC secondary control, it is not possible to show differences from the graphic point of view. Then, these differences will be presented in data table (Tables 2 and 3 ).

Table 2 shows the values $\sum_{j=1}^{1500} \Delta \omega_{i}(j)^{2}$ for each generator $i$, using frequency variation $\Delta \omega_{i}(t)$ with $i=1, \ldots, 10$, in the case of applying the traditional secondary control and for the case of applying the secondary MPC control to load variation on bar 18 in area 1 of EPS IEEE-39.

In this case, the average value for the 10 generators using the traditional secondary control is $2.12 \%$ higher than the average for the corresponding MPC secondary controller, being a great improvement of the behavior of the system when using MPC control. Also we have load variation on bar 22 (area 3) and load variation on bar 29 (area 4); results are presented in Table 3.

Table 3 shows that there is an improvement in each area at least of $2 \%$ higher than the average for the corresponding MPC secondary controller. Demonstrating the effectiveness of the control method which is independent of the selected area (by definition EPS IEEE-39) does not consider loads in area 2.

Figure 9(a) shows the power reference for generators that perform secondary control. Figure 9 (b) shows the additive power signal generated by the MPC, to support the secondary frequency control, which is small; however, its application achieves improved controller behavior. Figure 9(c) shows ACE signal, in response to varying load in area 1, also we see that the generator 10 (responsible for secondary control area 1 ) is the one that provides the most power between the four generators with secondary control. In addition, the expected convergence of (24) fails because the time intervals disposed for the load signal variation are small for this convergence.

Note that the works referenced in this paper [21, 25] perform applications in secondary MPC control using all the EPS machines, which is not reasonable due to economic and technical reasons, for example, higher cost of communication for all machines of system, absence of spinning reserve in a specific generator, and energy cost generator incompatible with the economic dispatch as secondary frequency controller. In this paper, however, the MPC is applied only in the machines which have secondary control in the area.

\section{Conclusions}

A frequency hierarchical supervisory control system is presented for an EPS, which improves the regulatory level through a new optimal signal support, keeping fixed the entire regulatory process system. The frequency control is applied to an electric power system IEEE-39, where the regulatory levels corresponding to the primary and secondary control were maintained, and an additive signal generated from a MPC algorithm was added to the traditional secondary control system, in order to optimize their performance. The results show the feasibility of this solution. There is an improvement of the system performance when using MPC control over the use of traditional secondary control; that is, the average squared frequency variation for the traditional secondary control was at least $2 \%$ higher than in the case of using the MPC secondary controller.

Note that the works referenced in this paper perform applications in secondary MPC control using all the EPS machines, which is not reasonable due to economic and technical reasons. In this paper, however, the MPC is applied only in the machines which already have secondary control. In future works, we will consider other characteristics of the units, such as their response speed, which will add an economic component to the analysis.

Finally, considering the measurement of energy quality, European standard UNE-EN 50160 requires variations in voltage frequency less than or equal to $1 \%$ for 10 seconds in $95 \%$ of the week. Then designing a controller that achieves an improvement at least $2 \%$ over the control of traditional frequency variations is a promising result.

\section{Conflict of Interests}

The authors declare that there is no conflict of interests regarding the publication of this paper. 


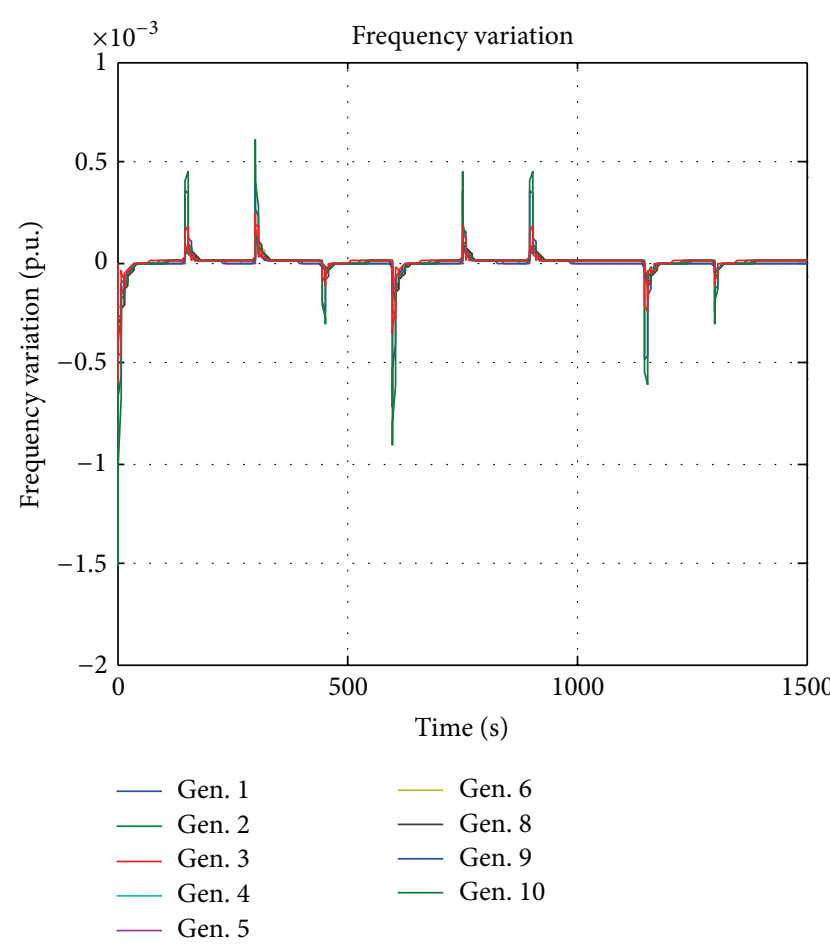

(a)

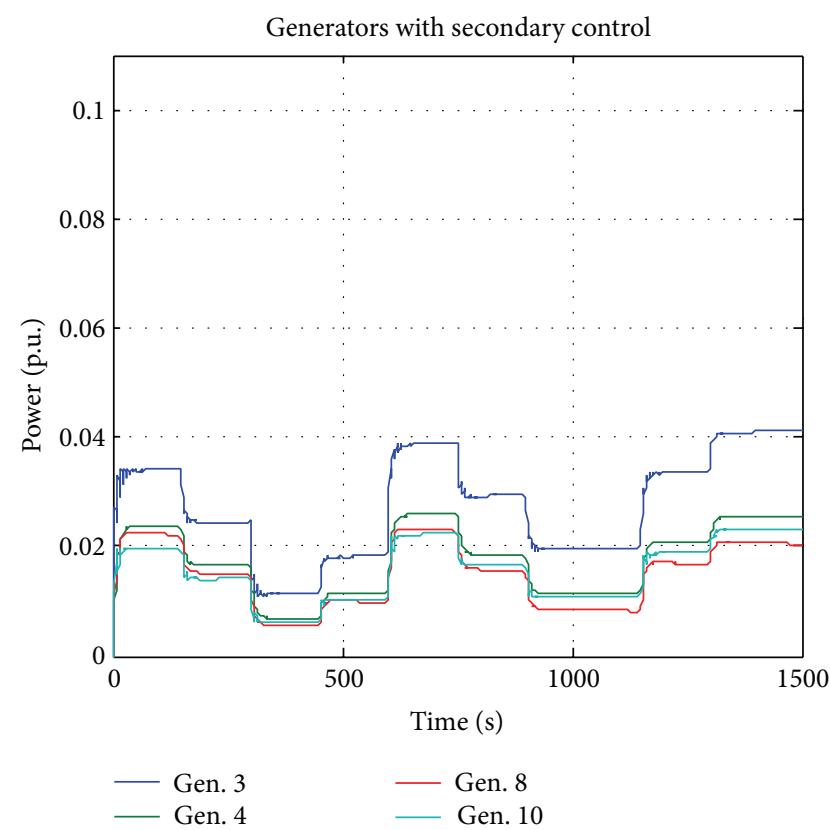

(c)

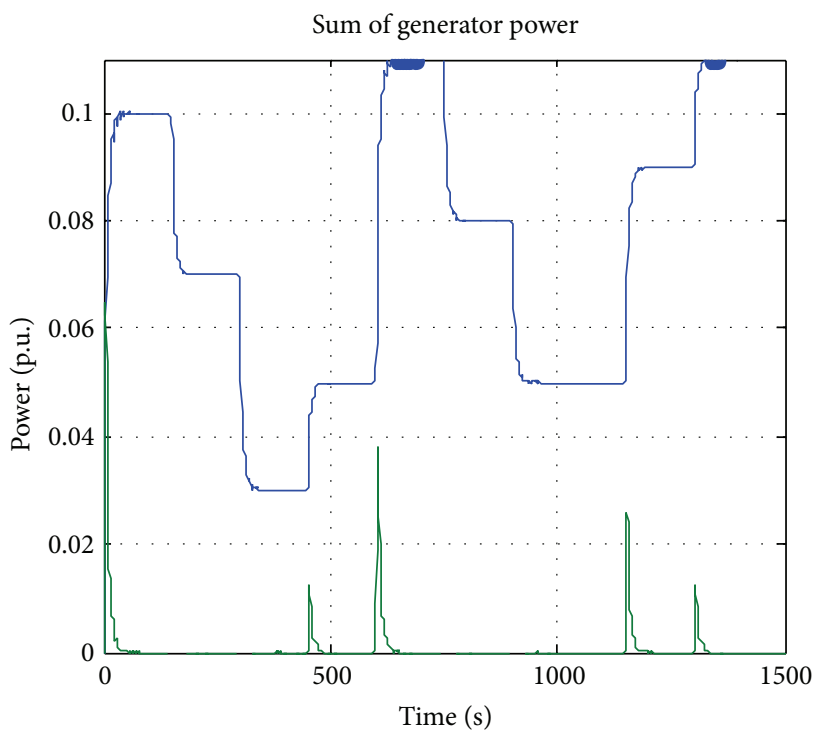

— Gen. 3, 4, 8, 10

— Gen. 1, 2, 5, 6, 7, 9

(b)

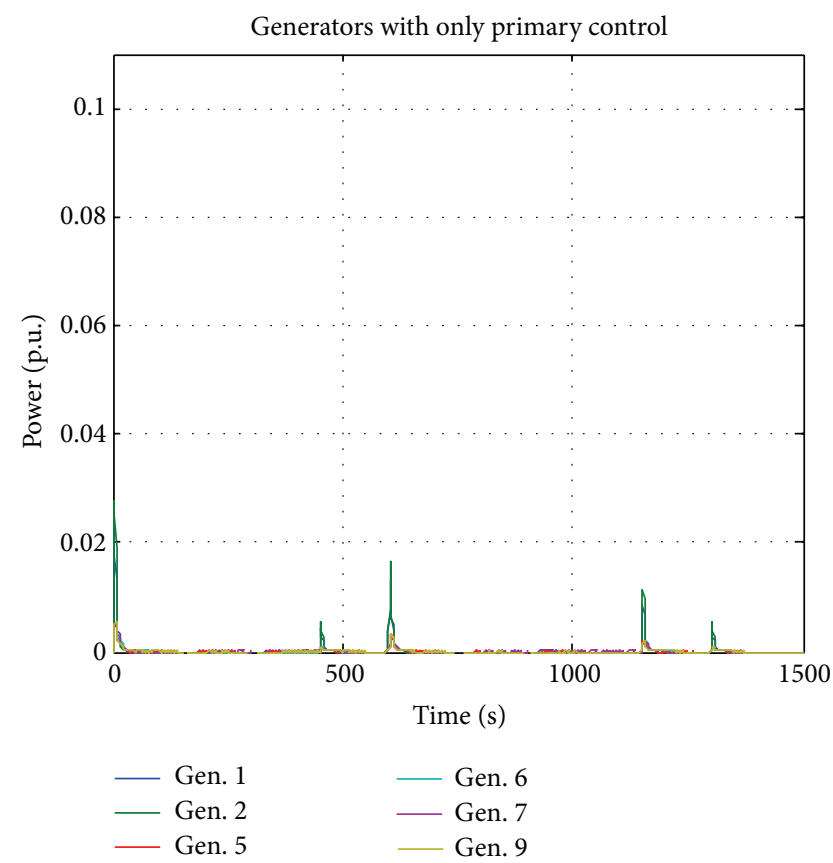

(d)

FIGURE 8: Secondary frequency MPC controller response.

TABLE 2: Accumulated squared frequency variation for secondary control.

\begin{tabular}{lcccccccccc}
\hline & \multicolumn{3}{c}{ Generators with secondary control } & \multicolumn{4}{c}{ Generators without secondary control } \\
Secondary control & Gen. 10 & Gen. 3 & Gen. 4 & Gen. 8 & Gen. 1 & Gen. 2 & Gen. 5 & $\begin{array}{c}\text { Gen. 6 } \\
\text { Gen. 7 }\end{array}$ & $\begin{array}{c}\text { Gen. 9 } \\
\end{array}$ \\
& $* 10^{-4}$ & $* 10^{-4}$ & $* 10^{-4}$ & $* 10^{-4}$ & $* 10^{-4}$ & $* 10^{-4}$ & $* 10^{-4}$ & $* 10^{-4}$ & $* 10^{-4}$ & $* 10^{-4}$ \\
\hline Traditional & 0.905 & 0.597 & 0.648 & 0.605 & 0.705 & 0.417 & 0.315 & 0.553 & 0.788 & 0.970 \\
MPC & 0.886 & 0.583 & 0.604 & 0.591 & 0.700 & 0.414 & 0.314 & 0.551 & 0.786 & 0.939 \\
\hline
\end{tabular}




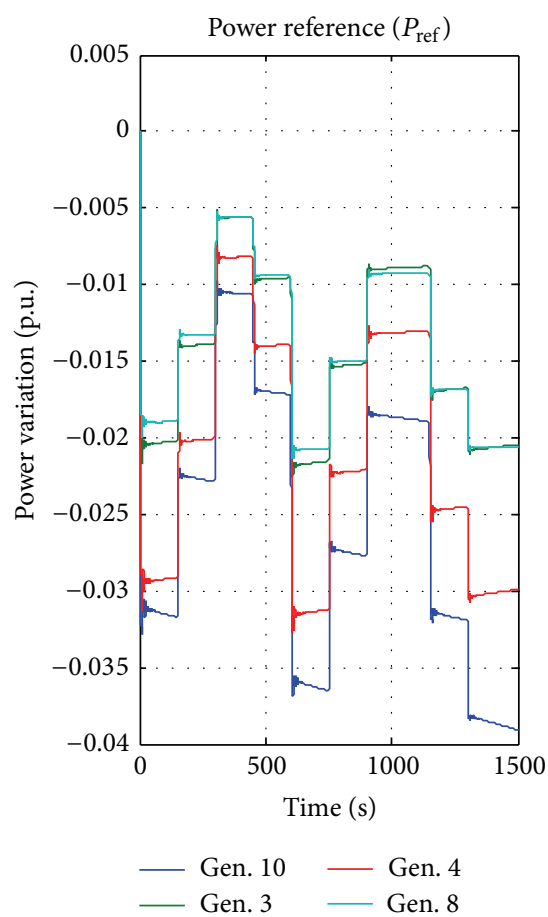

(a)

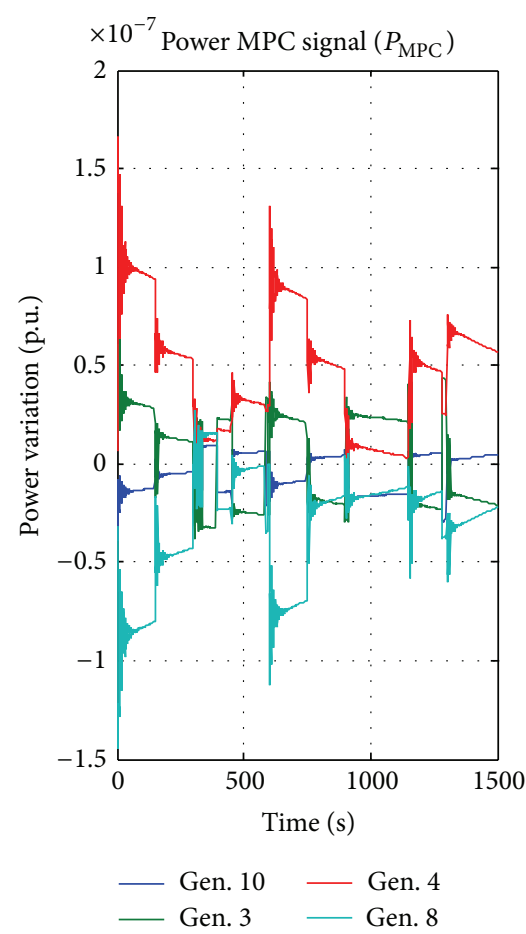

(b)

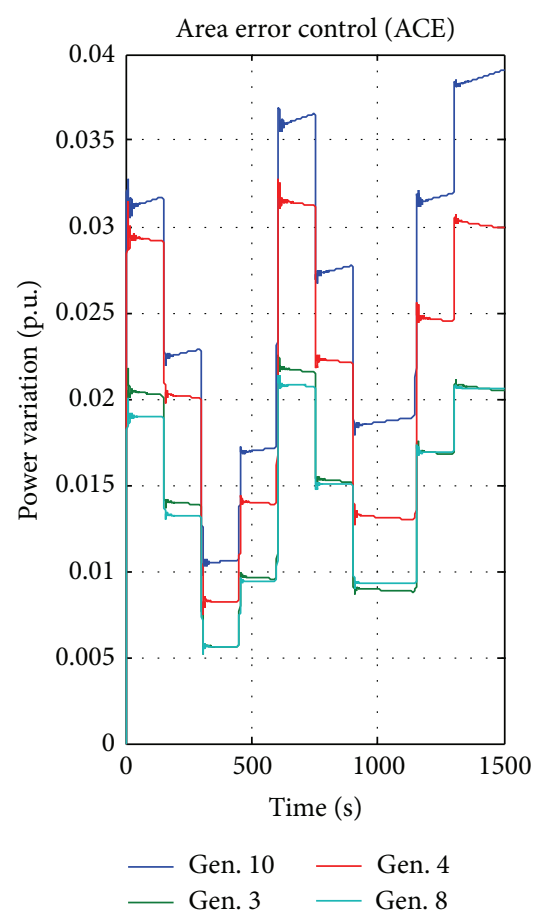

(c)

FIGURE 9: Power reference, additive MPC power, and ACE for generators performing secondary control.

TABLE 3: Improvement secondary control.

\begin{tabular}{lcccc}
\hline \multicolumn{2}{c}{ Place of load variation } & \multicolumn{2}{c}{ Secondary control } \\
Area & bar & Traditional mean & MPC mean & Improvement secondary MPC versus traditional \% \\
& 18 & 6.503 & 6.368 & 2.12 \\
\hline 1 & 22 & 7.053 & 6.908 & 2.06 \\
3 & 29 & 7.872 & 7.707 & 2.10 \\
\hline
\end{tabular}

\section{Acknowledgments}

This work has been supported by CONICYT, Chile, under Grant FB009 "Advanced Mining Technology Center." The first author would like to thank the support of CONICYT/FONDECYT Postdoctorado/(No. 3140604).

\section{References}

[1] P. Anderson and A. Fouad, Power System Control and Stability, IEEE Press, New York, NY, USA, 1993.

[2] P. Kundur, Power System Stability and Control, Power System Engineering Series, McGraw-Hill, New York, NY, USA, 1994.

[3] E. F. Camacho and C. Bordons, Model Predictive Control, Springer, 1999.

[4] D. Q. Mayne, J. B. Rawlings, C. V. Rao, and P. O. M. Scokaert, "Constrained model predictive control: stability and optimality," Automatica, vol. 36, no. 6, pp. 789-814, 2000.

[5] A. G. Beccuti, T. Geyer, and M. Morari, "A hybrid system approach to power systems voltage control," in Proceedings of the 44th IEEE Conference on Decision and Control, and the European Control Conference, pp. 6774-6779, Seville, Spain, December 2005 .

[6] M. Larsson, D. J. Hill, and G. Olsson, "Emergency voltage control using search and predictive control," International Journal of Electrical Power and Energy Systems, vol. 24, no. 2, pp. 121-130, 2002.

[7] B. Gong and I. Hiskens, "A stable finite horizon model predictive control for power system voltage collapse prevention," in Proceedings of the 50th IEEE Conference on Decision and Control and European Control Conference, pp. 7105-7110, Orlando, Fla, USA, 2011.

[8] M. Hajian, W. Rosehart, M. Glavic, H. Zareipour, and T. Van Cutsem, "Linearized power flow equations based predictive control of transmission voltages," in Proceedings of the 46th Hawaii International Conference on System Sciences, Hawaii, Hawaii, USA, 2013.

[9] L. Wang, H. Cheung, A. Hamlyn, and R. Cheung, "Model prediction adaptive control of inter-area oscillations in multigenerators power systems," in Proceedings of the IEEE Power and Energy Society General Meeting, pp. 1-7, Toronto, Canada, July 2009. 
[10] A. Tejada, "Efectos de las armónicas en los sistemas eléctricos," 2013, http:/www.mty.itesm.mx/decic/deptos/ie/profesores/allamas/cursos/ueee/armonicas/07Efectarm.PDF.

[11] G. Lemieux, "Power system harmonic resonance-a documented case," IEEE Transactions on Industry Applications, vol. 26, no. 3, pp. 483-488, 1990.

[12] E. J. Currence, J. E. Plizga, and H. N. Nelson, "Harmonic resonance at a medium-sized industrial plant," IEEE Transactions on Industry Applications, vol. 31, no. 4, pp. 682-690, 1995.

[13] G. T. Heydt, Electric Power Quality, West LaFayette: Stars in a Circle Publications, 1991.

[14] J. K. Phipps, J. P. Nelson, and P. K. Sen, "Power quality and harmonic distortion on distribution systems," IEEE Transactions on Industry Applications, vol. 30, no. 2, pp. 476-484, 1994.

[15] J. R. Linders, "Electric wave distortions: their hidden costs and containment," IEEE Transactions on Industry Applications, vol. 15, no. 5, pp. 458-471, 1979.

[16] H. Aggrawal, J. I. Leon, L. G. Franquelo, S. Kouro, P. Garg, and J. Rodríguez, "Model predictive control based selective harmonic mitigation technique for multilevel cascaded $\mathrm{H}$-bridge converters," in Proceedings of the 37th Annual Conference of the IEEE Industrial Electronics Society, pp. 4427-4432, Melbourne, Australia, November 2011.

[17] L. Garcia Franquelo, J. Nápoles, R. C. Portillio Guisado, J. I. Leon, and M. A. Aguirre, "A flexible selective harmonic mitigation technique to meet grid codes in three-level PWM converters," IEEE Transactions on Industrial Electronics, vol. 54, no. 6, pp. 3022-3029, 2007.

[18] T. H. Mohamed, H. Bevrani, A. A. Hassan, and T. Hiyama, "Decentralized model predictive based load frequency control in an interconnected power system," Energy Conversion and Management, vol. 52, no. 2, pp. 1208-1214, 2011.

[19] P. Hines, D. Jia, and S. Talukdar, "Distributed model predictive control for electric grids," in Proceedings of the Carnegie Mellon Transmission Conference, Pittsburgh, Pa, USA, 2004.

[20] S. Yin, S. Ding, A. Haghani, H. Hao, and P. Zhang, "A comparison study of basic data-driven fault diagnosis and process monitoring methods on the benchmark Tennessee Eastman process," Journal of Process Control, vol. 22, no. 9, pp. 1567-1581, 2012.

[21] A. N. Venkat, J. B. Rawlings, and S. J. Wright, "Stability and optimality of distributed model predictive control," in Proceedings of the 46th IEEE Conference on Decision and Control, and the European Control, pp. 6680-6685, Seville, Spain, December 2005.

[22] J. E. Cohen, "Cooperation and self-interest: pareto-inefficiency of Nash equilibria in finite random games," Proceedings of the National Academy of Sciences of the United States of America, vol. 95, no. 17, pp. 9724-9731, 1998.

[23] S. Yin, S. X. Ding, A. H. A. Sari, and H. Hao, "Data-driven monitoring for stochastic systems and its application on batch process," International Journal of Systems Science, vol. 44, no. 7, pp. 1366-1376, 2013.

[24] A. Damoiseaux, A. Jokic, L. Mircea et al., "Assessment of decentralized model predictive control techniques for power networks," in Proceedings of the 16th Power Systems Computation Conference, Glasgow, Scotland, 2008.

[25] R. M. Hermans, M. Lazar, and A. Jokić, "Distributed predictive control of the 7-machine CIGRÉ power system," in Proceedings of the American Control Conference (ACC '11), pp. 5225-5230, San Francisco, Calif, USA, July 2011.
[26] L. Xie and M. D. Ilić, "Model predictive economic/ environmental dispatch of power systems with intermittent resources," in Proceedings of the IEEE Power and Energy Society General Meeting (PES '09), pp. 1-6, IEEE, Pittsburgh, Pa, USA, July 2009.

[27] A. Ulbig, M. D. Galus, S. Chatzivasileiadis, and G. Andersson, "General frequency control with aggregated control reserve capacity from time-varying sources: The case of PHEVs," in Proceedings of the IREP Symposium-Bulk Power System Dynamics and Control-VIII (IREP '10), Buzios, Brazil, August 2010.

[28] T. Takagi and M. Sugeno, "Fuzzy identification of systems and its applications to modeling and control," IEEE Transactions on Systems, Man and Cybernetics, vol. 15, no. 1, pp. 116-132, 1985.

[29] J. Qiu, G. Feng, and J. Yang, "A new design of delay-dependent robust Ho filtering for discrete-time T-S fuzzy systems with time-varying delay," IEEE Transactions on Fuzzy Systems, vol. 17, no. 5, pp. 1044-1058, 2009.

[30] J. Qiu, G. Feng, and H. Gao, "Observer-based piecewise affine output feedback controller synthesis of continuous-time T-S fuzzy affine dynamic systems using quantized measurements," IEEE Transactions on Fuzzy Systems, vol. 20, no. 6, pp. 10461062, 2012.

[31] J. Qiu, G. Feng, and H. Gao, "Fuzzy-model-based piecewise $\mathscr{H} \infty$ static-output-feedback controller design for networked nonlinear systems," IEEE Transactions on Fuzzy Systems, vol. 18, no. 5, pp. 919-934, 2010.

[32] S. J. Qin and T. A. Badgwell, "An overview of nonlinear model predictive control applications," in Nonlinear Model Predictive Control, F. Allgöwer and A. Zheng, Eds., Birkhäuser, Basel, Switzerland, 2000. 


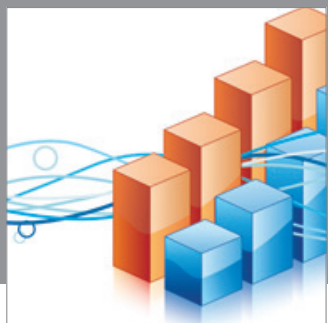

Advances in

Operations Research

mansans

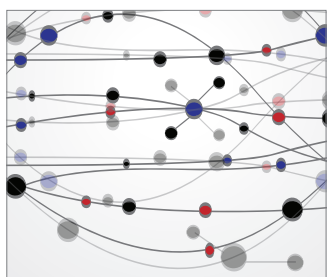

The Scientific World Journal
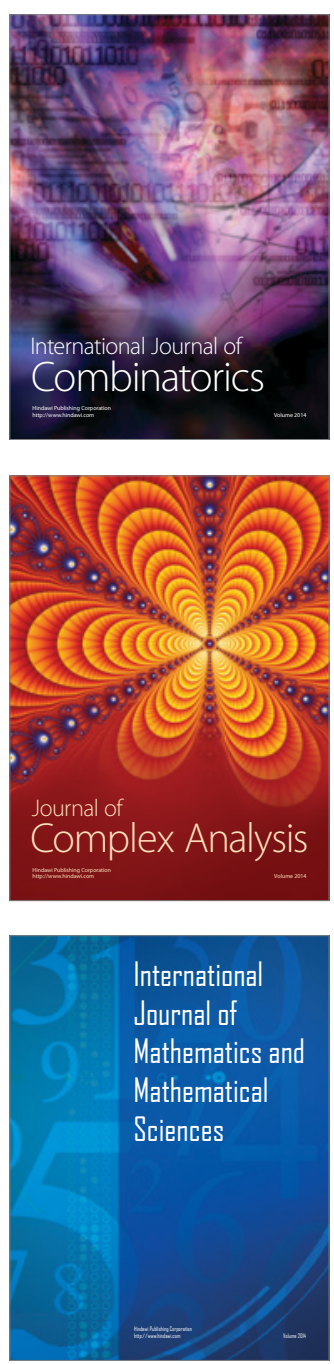
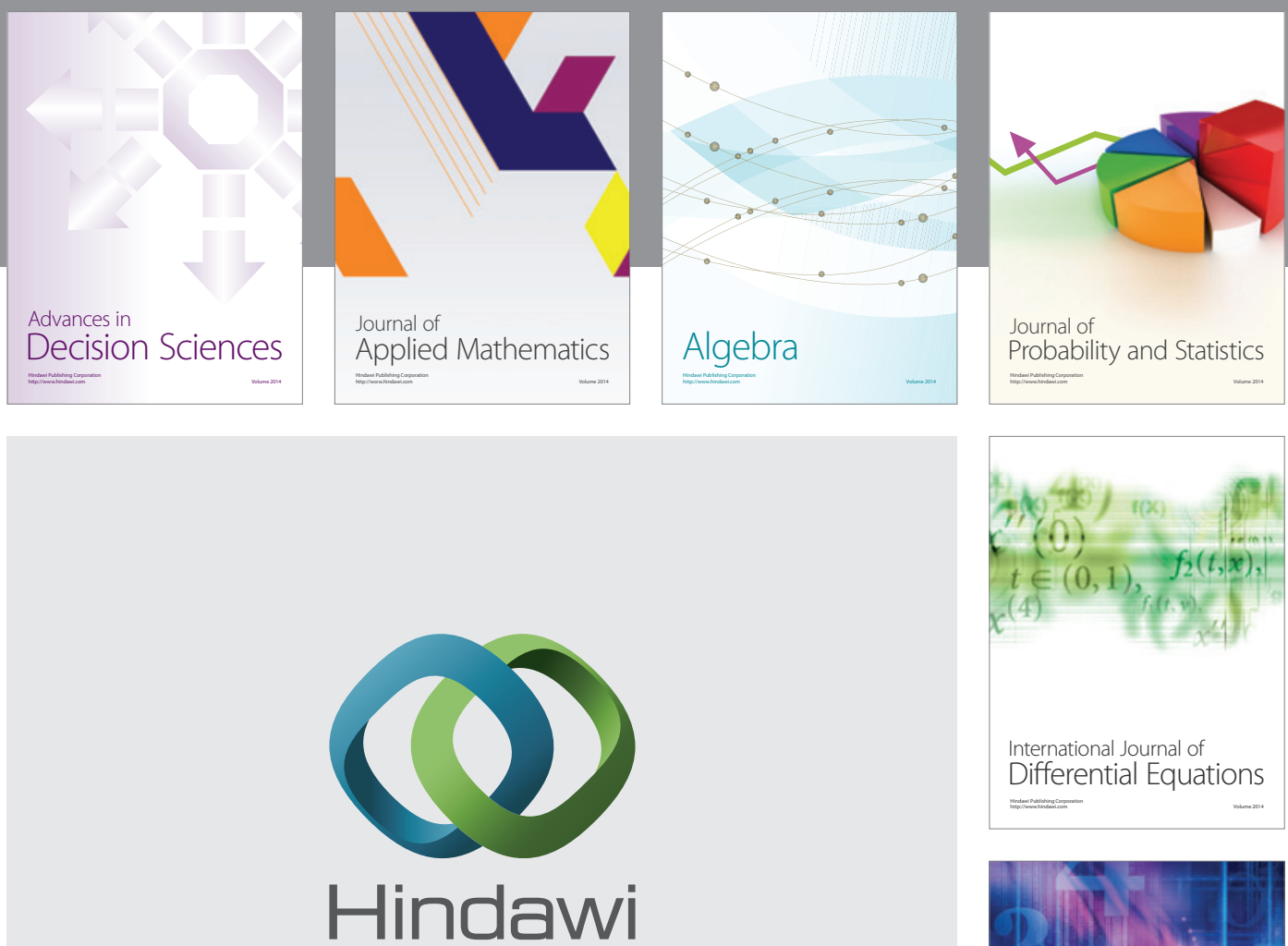

Submit your manuscripts at http://www.hindawi.com
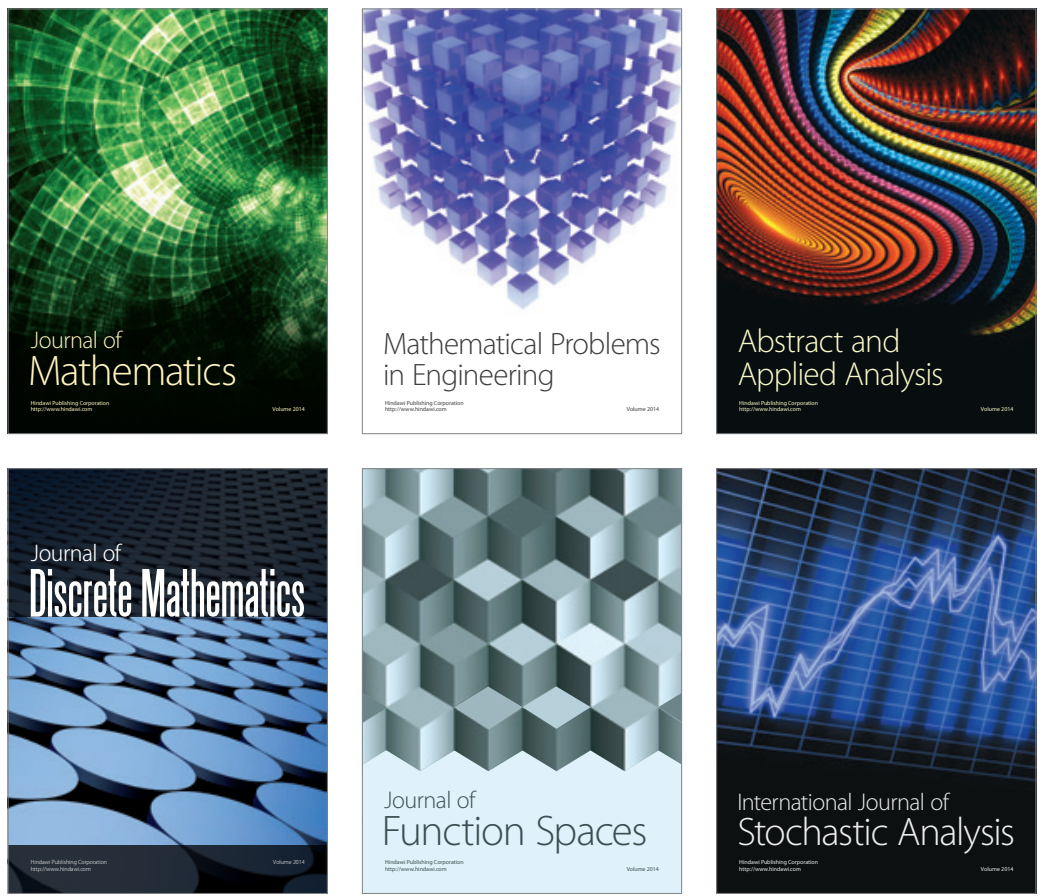

Journal of

Function Spaces

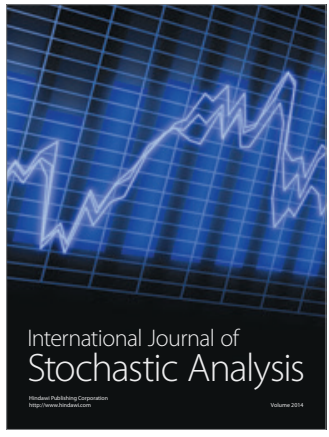

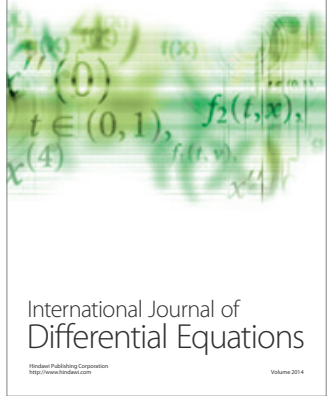
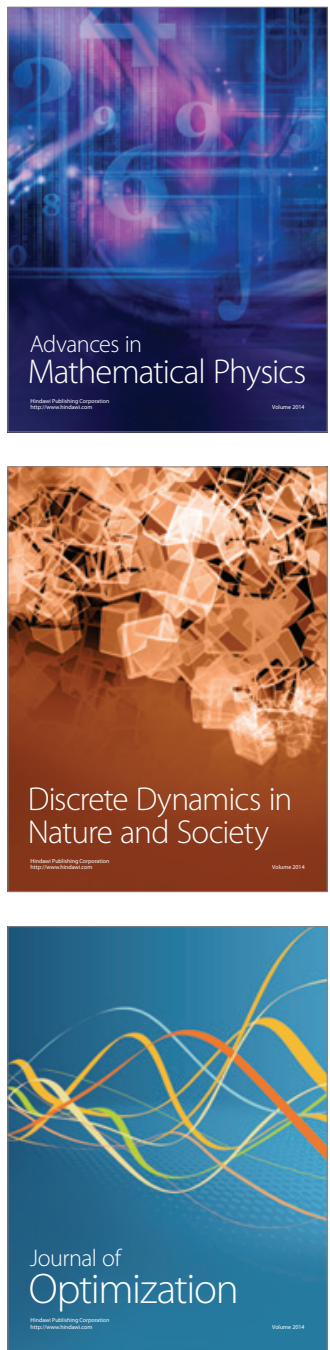